\title{
Comparative study of fat-suppression techniques for hip arthroplasty MR imaging
}

\section{Sébastien Molière, Jean-Philippe Dillenseger, Matthieu Ehlinger, Stéphane Kremer \& Guillaume Bierry}

\section{Skeletal Radiology}

Journal of the International Skeletal Society A Journal of Radiology, Pathology and Orthopedics

\section{ISSN 0364-2348}

Volume 46

Number 9

Skeletal Radiol (2017) 46:1209-1217 DOI 10.1007/s00256-017-2670-1

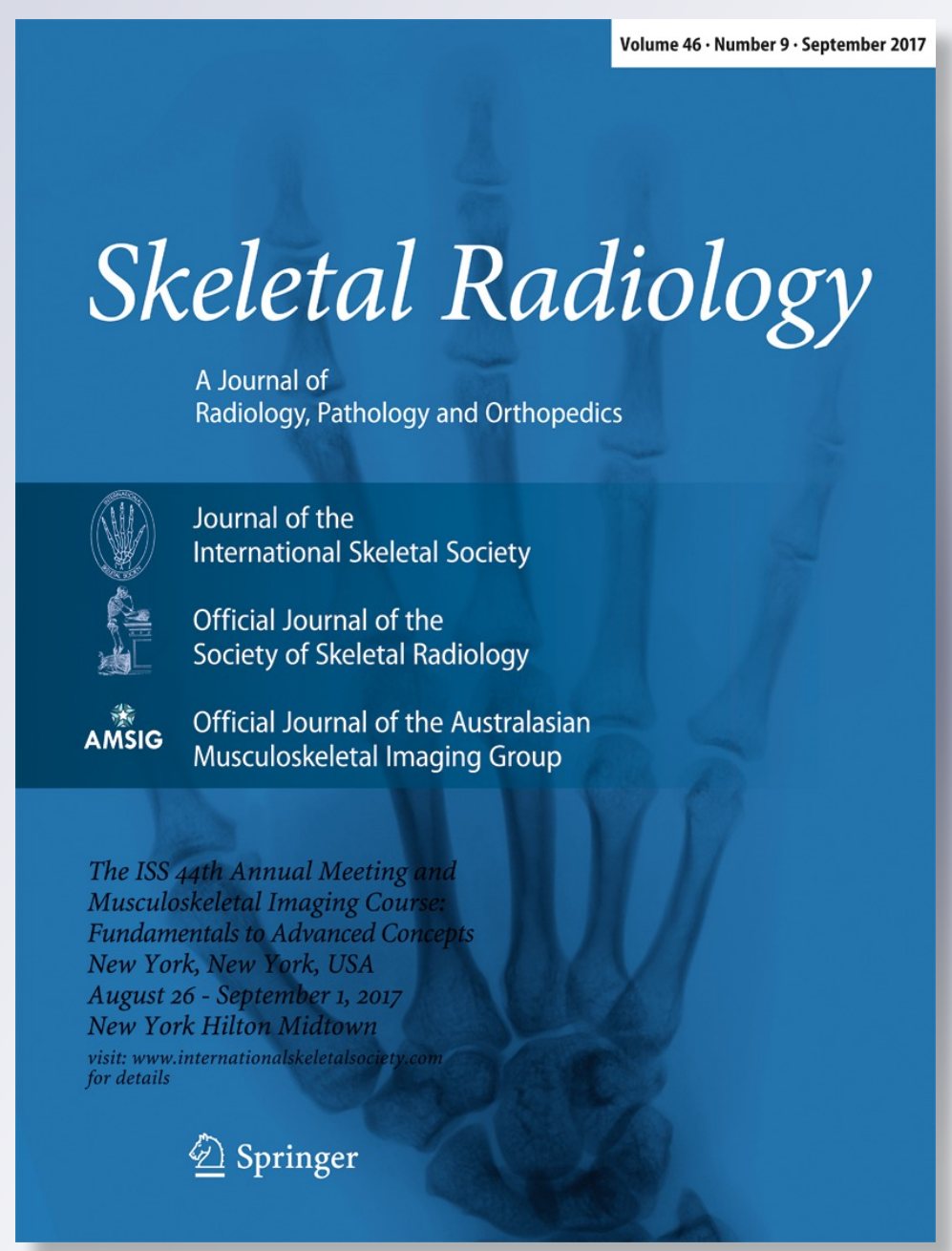

勿 Springer 
Your article is protected by copyright and all rights are held exclusively by ISS. This eoffprint is for personal use only and shall not be self-archived in electronic repositories. If you wish to self-archive your article, please use the accepted manuscript version for posting on your own website. You may further deposit the accepted manuscript version in any repository, provided it is only made publicly available 12 months after official publication or later and provided acknowledgement is given to the original source of publication and a link is inserted to the published article on Springer's website. The link must be accompanied by the following text: "The final publication is available at link.springer.com". 


\title{
Comparative study of fat-suppression techniques for hip arthroplasty MR imaging
}

\author{
Sébastien Molière $^{1} \cdot$ Jean-Philippe Dillenseger ${ }^{1,2}$ - Matthieu Ehlinger ${ }^{2,3}$. \\ Stéphane Kremer $^{1,2} \cdot$ Guillaume Bierry ${ }^{1,2}$
}

Received: 15 February 2017 /Revised: 18 April 2017 / Accepted: 2 May 2017 /Published online: 24 May 2017

(C) ISS 2017

\begin{abstract}
Objectives The goal of this study was to evaluate different fatsuppressed fluid-sensitive sequences in association with different metal artifacts reduction techniques (MARS) to determine which combination allows better fat suppression around metallic hip implants.

Methods An experimental study using an MRI fat-water phantom quantitatively evaluated contrast shift induced by metallic hip implant for different fat-suppression techniques and MARS. Then a clinical study with patients addressed to MRI unit for painful hip prosthesis compared these techniques in terms of fat suppression quality and diagnosis confidence. Results Among sequences without MARS, both T2 Dixon and short tau inversion recuperation (STIR) had significantly lower contrast shift $(p<0.05)$, Dixon offering the best fat suppression. Adding MARS (view-angle tilting or sliceencoding for metal artifact correction (SEMAC)) to STIR gave better results than Dixon alone, and also better than SPAIR and fat saturation with MARS $(p<0.05)$. There were no statistically significant differences between STIR with view-angle tilting and STIR with SEMAC in terms of fat suppression quality.

Conclusions STIR sequence is the preferred fluid-sensitive MR sequence in patients with metal implant. In combination
\end{abstract}

Guillaume Bierry

guillaume.bierry@chru-strasbourg.fr

1 Imaging Department, University Hospital of Strasbourg, 1 Avenue Moliere, 67098 Strasbourg, France

2 ICube UMR 7357, University of Strasbourg, Strasbourg, France

3 Orthopaedic Department, University Hospital of Strasbourg, Strasbourg, France with MARS (view-angle tilting or SEMAC), STIR appears to be the best option for high-quality fat suppression.

Keywords MRI $\cdot$ Hip $\cdot$ Prosthesis $\cdot$ Fat suppression $\cdot$ Metal artifact reduction sequences

$\begin{array}{ll}\text { Abbreviations } \\ \text { STIR } & \text { Short tau inversion recovery } \\ \text { SEMAC } & \text { Slice-encoding for metal artifact correction } \\ \text { SPAIR } & \text { Spectral attenuated inversion recovery } \\ \text { FS } & \text { (Spectrally selective) fat suppression } \\ \text { WE } & \text { Water excitation }\end{array}$

\section{Introduction}

Hip arthroplasty is an increasingly frequent surgical procedure in the aging population [1]. Early detection and management of implant complications are key for long-term prognosis [2]. Along with other modalities, MRI is nowadays recognized as a powerful tool for the assessment of patients with painful prosthesis, especially for evaluation of soft tissues and bone marrow $[3,4]$.

Its main drawback, in this setting, remains a potential serious deterioration of MR images, due to local disturbance of magnetic field by ferromagnetic implants, resulting in artifacts such as signal loss, signal pile-up, and both in-plane and through-plane distortion, by interfering with read-out frequency encoding and section-selection gradient $[5,6]$. Several metallic artifact reducing sequences (MARS) exist, from simple modifications of spin echo-based sequences - such as increased slice-selection gradient and larger readout bandwidths [7] — to more refined techniques such as view-angle tilting and 
slice-encoding for metal artifact correction technique (SEMAC) $[8,9]$.

The second important consequence of local magnetic field offset is the failure of fat signal suppression [10]. Fatsuppression techniques rely on the chemical shift between protons in water and protons in fat, the latter precessing at a slightly lower frequency. Spectrally selective fat saturation (FS) and spectral attenuated inversion recovery (SPAIR) begin with fat or water-specific frequency selection, making them prone to artifacts induced by magnetic field inhomogeneity [11]. Water excitation (WE) techniques use fat and water phase period (about $2.3 \mathrm{~ms}$ at 1.5 Tesla) induced by chemical shift, making them prone to artifacts induced by magnetic field inhomogeneity too [12]. In the Dixon technique, separation of water and fat signal is postponed during the reconstruction, and multipoints Dixon sequences have shown encouraging resilience to magnetic field inhomogeneity [13]. Short tau inversion recovery (STIR) sequence relies on the shorter T1 relaxation time of the fat tissue compared to that of water, making this sequence relatively insensitive to magnetic field inhomogeneity [10].

Many studies have specifically addressed the problem of metal-induced distortion artifacts [14-16], but experimental studies evaluating fat-suppression efficiency around metallic implants are scarce, despite the potential clinical implications for evaluation of implant loosening, infection, and pseudotumors especially in T2-weighted sequences [17].

The goal of this study was to systematically evaluate the majority of available fluid-sensitive sequences fat-suppression techniques (FS, WE, Dixon, or SPAIR on fast spin echo T2weighted sequences; STIR sequence) in association with different metal artifacts reduction techniques, to determine which combination allows better fat suppression around metallic hip implants. The first part is an experimental study using an MRI fat-water phantom including a metallic hip implant. The second part is a clinical study with patients referred for MRI of a painful hip prosthesis to illustrate the clinical significance of high-quality fat suppression.

\section{Materials and methods}

\section{Experimental study}

\section{Phantoms}

A metal-on-metal total hip prosthesis (Zimmer, Metasul, Warsaw, IN, USA) composed of a titanium femoral stem coated with hydroxyapatitis, a cobalt-chrome (Cr-Co) 28$\mathrm{mm}$ femoral head and acetabular polyethylene insert with cobalt-chrome metal inlay Metasul, measuring $196 \times 68 \times$ $50 \mathrm{~mm}$ (length $\times$ width $\times$ height) was put in a rectangular tank of agarose gel $8 \%$. A metal-on-metal implant was chosen because it is still largely present worldwide. Twenty-two polystyrene culture tubes (Thomas \& Betts, Memphis, TN, USA), of 13-mm diameter and 100-mm length, containing peanut oil, to mimic fat, were placed around the implant following a grid and covering a $20 \times$ $35-\mathrm{cm}$ rectangular area (Fig. 1). Besides, a control phantom was build with agarose gel and oil tubes, without metallic implant (Fig. 1).

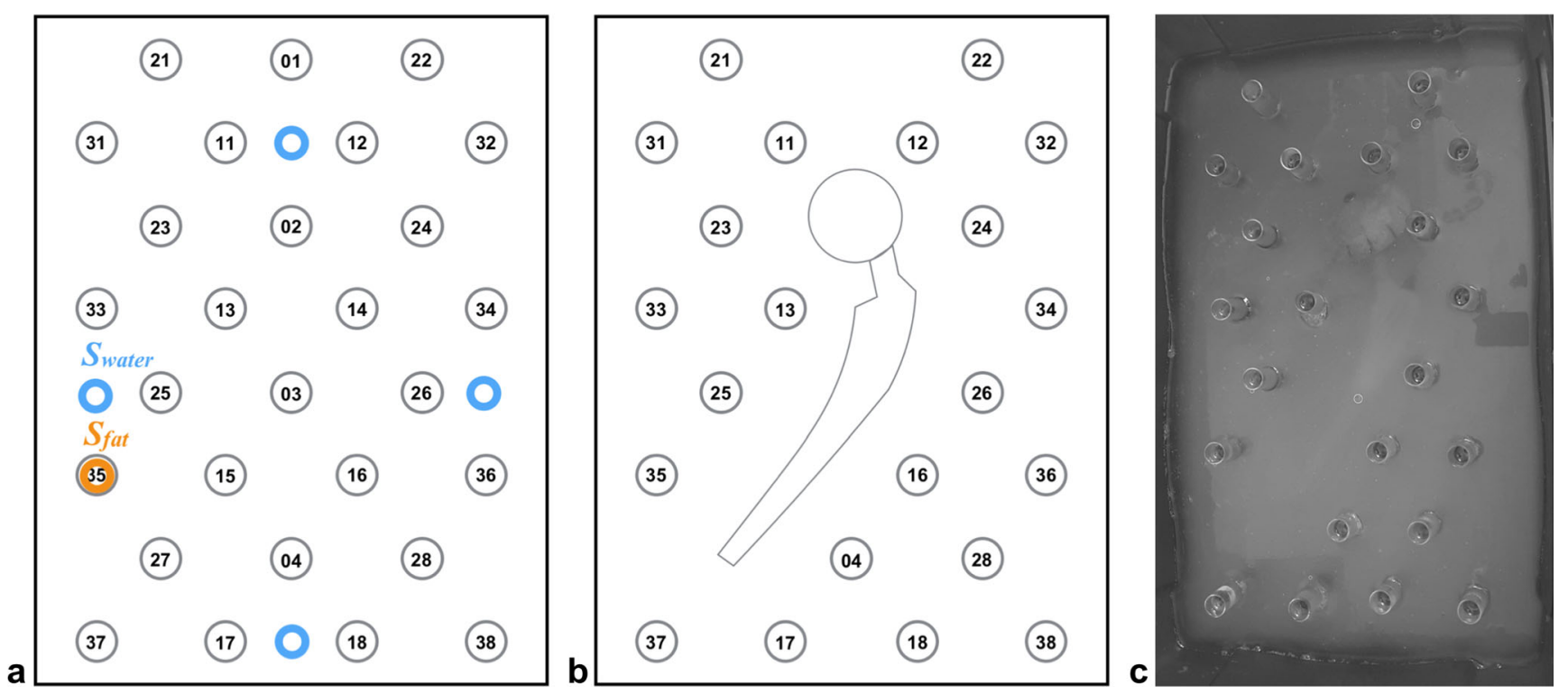

Fig. 1 Schematic design of phantoms without (a) and with (b) metal prosthesis, and photograph of the phantom (c). Each circle in a and $\mathbf{b}$ represents a fat tube. Sfat represents regions of interest (ROI) in fat tube no. 35, while Swater represent ROI in agarose gel 


\section{MR parameters}

Phantoms were scanned in a clinical 1.5-T MRI system (Magnetom Aera, Siemens, Erlangen, Germany) using the constructor anterior body eight-channel coil and posterior spine coil (eight active channels). They were placed longitudinally on the MRI table, the long axis of the prosthesis following the same direction as $\mathrm{B}_{0}$ field, which presents a $45-\mathrm{cm}$ diameter of spherical volume (DSV), approximately at the magnetic isocenter of the MRI unit. For each phantom were acquired routine coronal STIR images and routine coronal T2 fast spin echo (FSE) images with spectrally selective fat suppression (FS), SPAIR; WE, and Dixon (Table 1). STIR, FSE T2 FS, and FSE T2 SPAIR sequences were then acquired with view-angle tilting and SEMAC.

To avoid confounding factors, the same geometrical parameters (FOV, matrix, slice thickness) and readout bandwidth $\left(\mathrm{BW}_{\mathrm{r}}\right)$ were used for each acquisition (Table 1). A $35 \times 35-\mathrm{cm}$ field of view was chosen to fully cover the phantom and correspond to a routine hip MRI examination. Following MARS parameters were chosen: VAT $100 \%$ and eight slice-encoding steps for SEMAC.

\section{Quantitative analysis}

Data were analyzed by a fellow-trained radiologist (SM), blinded to the type of sequences, using OsiriX software. To evaluate intra-rater agreement, measurement of all datasets was repeated by the same observer after a 2 -week interval. For each sequence, the same slice through the long axis of the implant was analyzed. A 5-mm-diameter circular region of interest (ROI) was placed in each tube of fat and four circular ROIs were placed in the agarose gel (Fig. 1). If the circular ROI did not fit in the tube because of major geometrical distortion artifact, the tube was excluded from the analysis.

Given that direct comparison of signal intensities between different acquisitions is not relevant in MRI, we calculate for each tube a normalized fat-water contrast [18], which is independent of any arbitrary image scale factor, with the following formula:

$\mathrm{C}=\frac{\left|S_{f a t}-S_{\text {water }}\right|}{S_{f a t}+S_{\text {water }}}$

where $S_{\text {fat }}$ is the average signal value in the fat tube ROI and $S_{\text {water }}$ the average signal value in the agarose ROIs.

The shift in fat-water contrast induced by the metallic implant in the fat-suppressed sequences was evaluated for each tube with following the formula:

Shift $_{R O I}=1-\frac{C_{\text {prosthesis }}}{C_{\text {ref }}}$

$C_{\text {prosthesis }}$ is the contrast of the tube in the prosthesis phantom and $C_{\text {ref }}$ the contrast of the same tube in the control

Table 1 MRI parameters

\begin{tabular}{|c|c|c|c|c|c|c|c|c|c|c|}
\hline FSE sequences & BWr (hz/px) & FOV (mm) & Matrix & Slice thickness $(\mathrm{mm})$ & $\mathrm{TE}(\mathrm{ms})$ & $\mathrm{TR}(\mathrm{ms})$ & TI (ms) & ETL & FA & Sequence duration \\
\hline \multicolumn{11}{|l|}{ Experimental study } \\
\hline $\mathrm{T} 2 \mathrm{FS}$ & 300 & 350 & 512 & 3.5 & 66 & 2960 & - & 12 & 150 & $2 \mathrm{~min}, 33 \mathrm{~s}$ \\
\hline STIR & 300 & 350 & 512 & 3.5 & 66 & 5560 & 150 & 12 & 150 & $5 \mathrm{~min}, 15 \mathrm{~s}$ \\
\hline T2 SPAIR & 300 & 350 & 512 & 3.5 & 109 & 4690 & - & 12 & 150 & $4 \mathrm{~min}, 8 \mathrm{~s}$ \\
\hline T2 WE & 300 & 350 & 512 & 3.5 & 66 & 2960 & - & 12 & 150 & $1 \mathrm{~min}, 45 \mathrm{~s}$ \\
\hline T2 Dixon & 300 & 350 & 512 & 3.5 & - & 4190 & - & 12 & 150 & $4 \mathrm{~min}, 30 \mathrm{~s}$ \\
\hline T2 FS VAT & 300 & 350 & 512 & 3.5 & 49 & 2800 & - & 12 & 150 & $3 \mathrm{~min}, 36 \mathrm{~s}$ \\
\hline STIR VAT & 300 & 350 & 512 & 3.5 & 67 & 5560 & 150 & 12 & 150 & $5 \mathrm{~min}, 15 \mathrm{~s}$ \\
\hline T2 SPAIR VAT & 300 & 350 & 512 & 3.5 & 49 & 4010 & - & 12 & 150 & $5 \mathrm{~min}, 25 \mathrm{~s}$ \\
\hline T2 FS SEMAC & 300 & 350 & 512 & 3.5 & 78 & 7870 & - & 12 & 150 & $13 \mathrm{~min}, 39 \mathrm{~s}$ \\
\hline STIR SEMAC & 300 & 350 & 512 & 3.5 & 27 & 7030 & 150 & 12 & 150 & $10 \mathrm{~min}, 13 \mathrm{~s}$ \\
\hline T2 SPAIR SEMAC & 300 & 350 & 512 & 3.5 & 46 & 4010 & - & 12 & 150 & $44 \min , 33 \mathrm{~s}$ \\
\hline \multicolumn{11}{|l|}{ Clinical study } \\
\hline Coronal SE T1 & 300 & $210 \times 210$ & $480 \times 640$ & 3.5 & 11 & 420 & - & 3 & 150 & $2 \mathrm{~min}, 46 \mathrm{~s}$ \\
\hline Coronal T2 FS VAT & 300 & $200 \times 200$ & $576 \times 784$ & 3 & 60 & 2800 & - & 9 & 150 & $3 \mathrm{~min}, 52 \mathrm{~s}$ \\
\hline Coronal STIR & 300 & $200 \times 200$ & $480 \times 640$ & 3.5 & 66 & 5560 & 150 & 12 & 150 & $5 \mathrm{~min}, 15 \mathrm{~s}$ \\
\hline Coronal STIR VAT & 300 & $200 \times 200$ & $480 \times 640$ & 3.5 & 66 & 5560 & 150 & 12 & 150 & $5 \mathrm{~min}, 15 \mathrm{~s}$ \\
\hline Axial STIR VAT & 300 & $200 \times 200$ & $480 \times 640$ & 3.5 & 66 & 5560 & 150 & 12 & 150 & $5 \mathrm{~min}, 15 \mathrm{~s}$ \\
\hline Sagittal STIR VAT & 300 & $200 \times 200$ & $480 \times 640$ & 3.5 & 66 & 5560 & 150 & 12 & 150 & $5 \mathrm{~min}, 15 \mathrm{~s}$ \\
\hline
\end{tabular}

$B W r$ readout bandwidth, $F O V$ field of view, $T E$ echo time, $T R$ repetition time, $T I$ inversion time, $E T L$ echo train length, $F A$ flip angle 
phantom (without implant). Mean contrast shift was calculated for the whole-phantom and for the peri-acetabular regions (tube nos. 11, 12, 13, 23, 24).

\section{Clinical study}

\section{Patients}

From January 2016 to June 2016, eight consecutive patients (mean age 63 years, range, 51-78 years) referred to the MRI unit for exploration of a painful metal-on-metal hip prosthesis were prospectively included. The study was conducted in compliance with ethical standards and all patients gave written informed consent. The study group consisted of four males (mean age 63 years, range, 54-68 years) and four females (mean age 63 years, range, 51-78 years). Average time since implantation was 7 years (range, $1-12$ years).

\section{MR protocol}

Patients were scanned on a clinical 1.5-T MRI system (Magnetom Aera, Siemens), using a torso eight-channel coil combined with a spine coil.

MR protocol included the following sequences: coronal SE T1-weighted; coronal FSE FS T2-weighted with view-angle tilting; coronal STIR; coronal, sagittal, and axial STIR with view-angle tilting (Table 1). T2 WE and T2 Dixon sequences were not performed, as they cannot be associated with MARS; STIR with SEMAC was not performed due to a too long examination time.
For each patient, abnormal findings were reported in consensus by two observers (GB with 15 years of experience in musculoskeletal radiology and SM with 5 years of experience in musculoskeletal radiology) using all available series and following a binary checklist: presence or not of periprosthetic bone marrow edema, joint effusion, extraarticular fluid collection or pseudo-tumor with mention of its communication with the hip joint, high-grade tendon tear.

\section{Qualitative analysis}

On each sequence, two independent observers (GB, SM) assessed fat-suppression homogeneity using a three-grade scale $(0=$ severe fat suppression inhomogeneity, $1=$ mild inhomogeneity only impairing analysis of the periprosthetic region, $2=$ no visible inhomogeneity) and diagnosis confidence in the evaluation of the previously listed abnormalities using on a three-grade scale $(0=$ no diagnosis possible due to major distortions, 1 = probable lesion, but a pseudo-lesion due to failure of fat suppression is not excluded, intermediate confidence $2=$ certain lesion, high confidence).

Inter-reader agreement was calculated, and in case of discrepancy consensus reading was retained for further analysis.

\section{Statistical analysis}

Data were analyzed using SPSS (v23, IBM, USA). A $p$ value inferior to 0.05 was considered as statistically significant.

For the experimental study, an intrarater reliability analysis using the intraclass coefficient correlation (ICC) was performed. Then, contrast shift was compared between each
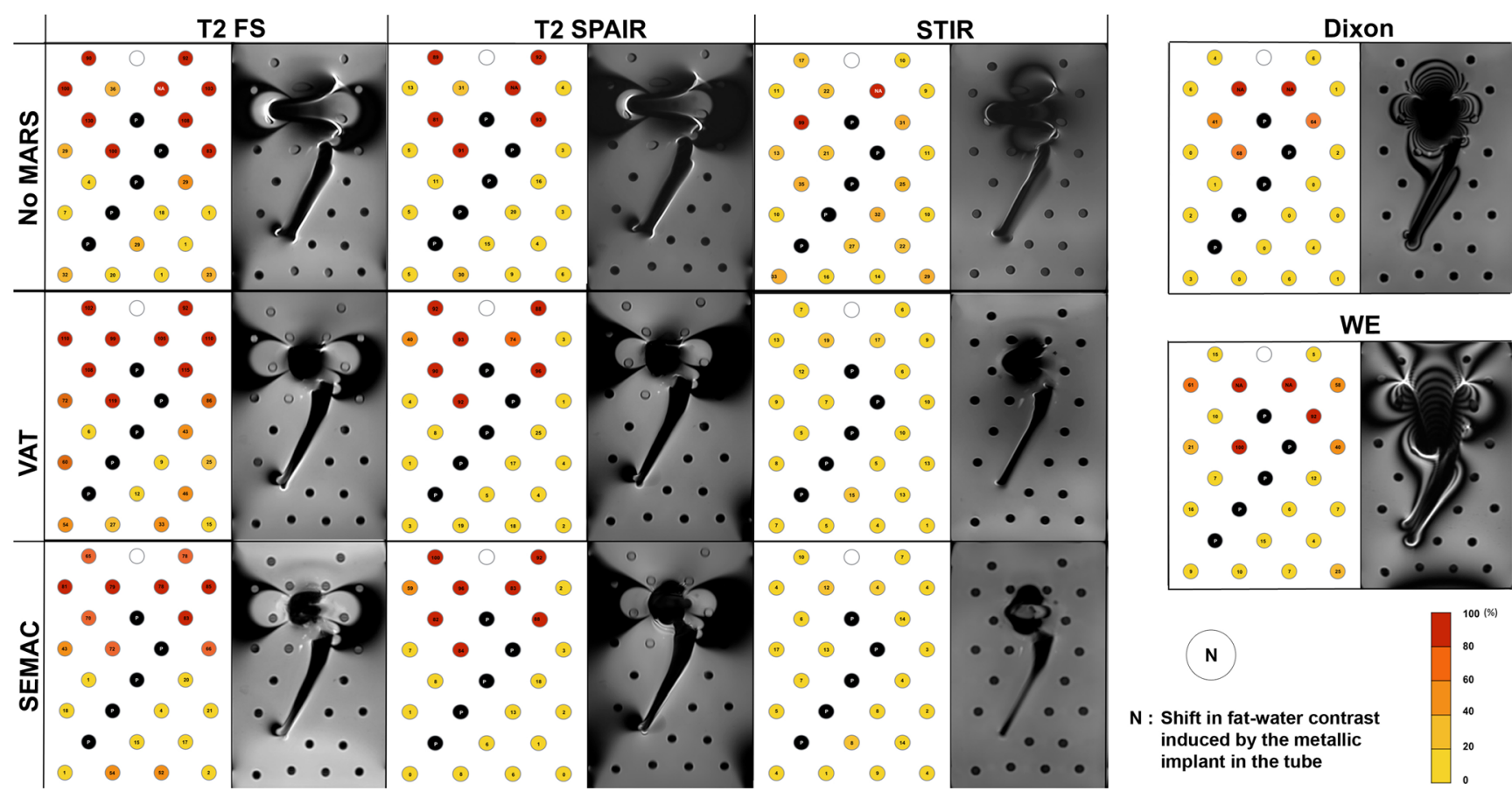

Fig. 2 MR images of the prosthesis phantom with different sequences and color-coded graphical representation of corresponding contrast shift 
sequence using analysis of variance with Tukey post hoc tests. Wilcoxon signed-rank test for paired values was used to compare sequences before and after MARS application.

For the clinical study, an interrater reliability analysis using the Kappa statistic was performed [19]. Fat-suppression homogeneity and diagnosis confidence were then compared for STIR, T2 FS view-angle tilting and STIR view-angle tilting sequences using analysis of variance with Tukey post hoc tests.

\section{Results}

\section{Experimental study}

Intra-rater agreement was ICC $=0.83(p<0.001), 95 \%$ CI (0.72 to 0.97$)$.

Among sequences without MARS, T2 Dixon and STIR had the lowest contrast shift (Fig. 2) (Table 2). Nevertheless, statistically significant differences were only present between STIR and T2 FS ( $p=0.01)$, and between T2 Dixon and T2 FS $(p=0.003)$ for the whole phantom. No differences were observed for periacetabular contrast shift between the sequences.

Fat suppression was poorer around the acetabular component, with periacetabular contrast shift being significantly higher for all sequences in comparison to whole-phantom contrast shift (Table 2).

Among sequences with MARS, STIR with view-angle tilting had significantly lower contrast shift than T2 FS with view-angle tilting for both whole-phantom and periacetabular region $(p=<0.0001)$, and also lower than T2 SPAIR with view-angle tilting for both whole-phantom and periacetabular region ( $p=0.004$ and $<0.0001$, respectively).

STIR with SEMAC had significantly lower contrast shift than T2 FS SEMAC for both whole-phantom and periacetabular region $(p=<0.0001)$, and also lower than T2 SPAIR SEMAC for both whole-phantom and periacetabular region $(p<0.0001)$.

STIR-based sequences had the lower contrast shift in the periacetabular region compared to other fluid-sensitive techniques.

Interestingly, application of view-angle tilting or SEMAC only improved significantly the STIR sequence, and only for whole phantom, not for periacetabular region (Table 3). In addition, there were no statistically significant differences between STIR with view-angle tilting and STIR with SEMAC (Table 3).

Artifact size - including both geometrical distortion and fat suppression inhomogeneity-was significantly reduced by combination of STIR and MARS (Fig. 3).

\section{Clinical study}

Three patients showed intra-articular fluid, one with extraarticular pseudo-tumor communicating with the articular 
Table 3 Experimental study: comparative results of contrast shift for T2 FS, T2 SPAIR and STIR sequences without MARS (w/o), with view-angle tilting (VAT) and with SEMAC (Wilcoxon signed-rank test) for whole-phantom (WP) and periacetabular regions $(\mathrm{PaR})$

\begin{tabular}{lllll}
\hline & & VAT versus w $/ 0$ & SEMAC versus w/o & VAT versus SEMAC \\
\hline \multirow{2}{*}{ T2 FS } & WP & 0.81 & 0.55 & 0.32 \\
\multirow{3}{*}{ T2 SPAIR } & PaR & 0.30 & 0.54 & 0.44 \\
& WP & 0.85 & 0.91 & 0.94 \\
\multirow{2}{*}{ STIR } & PaR & 0.51 & 0.60 & 0.65 \\
& WP & $0.003^{\mathrm{a}}$ & $0.001^{\mathrm{a}}$ & 0.18 \\
& $\mathrm{PaR}$ & 0.07 & 0.07 & 0.45 \\
\hline
\end{tabular}

${ }^{\mathrm{a}}$ Indicates a statistically significant difference $(p<0.05)$ space, and one with periprosthesis bone marrow edema consistent with prosthesis loosening (Table 4) (Figs. 4 and 5).

The interrater reliability for the raters was found to be Kappa $=0.94$ (almost perfect agreement) $(p<0.001), 95 \%$ CI $(0.94,0.90)$ for fat-suppression homogeneity, and Kappa $=0.90$ (almost perfect agreement) $(p<0.001), 95 \%$ CI $(0.92,0.88)$ for diagnosis confidence.

Fat-suppression homogeneity and diagnosis confidence were significantly poorer in T2 FS with view-angle tilting compared to conventional STIR, and were significantly improved by the application of view-angle tilting on the STIR sequence (Table 4) (Figs. 4 and 5).

\section{Discussion}

This study demonstrated experimentally that STIR and Dixon techniques better suppress fat signals in the presence of a metallic prosthesis compared to FS T2, SPAIR T2, and WE sequences. Dixon sequence tended to show better wholephantom fat suppression but was severely impaired by

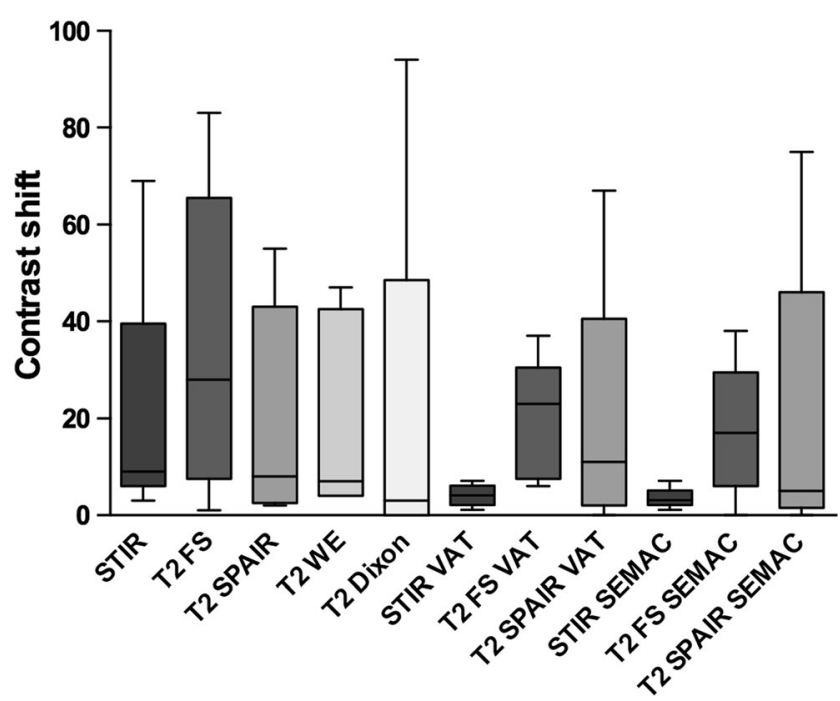

Fig. 3 Box plot representing whole-phantom contrast shift for each sequence of the experimental study artifacts in the periprosthetic region, as well as STIR sequence without MARS.

Adding metal artifact reduction (view-angle tilting and SEMAC) to STIR sequence significantly improved fatsuppression homogeneity on both experimental and clinical studies, by decreasing the periprosthetic voids and distortions.

View-angle tilting is an imaging technique in which an additional readout gradient is applied along the sectionselective direction, thus producing an additional phase. This phase offsets unwanted phase accumulation caused by field inhomogeneity, causing pixels shearing and thus compensating the pixel shift in the readout direction which reduce inplane distortion with no additional scan time [7, 8]. SEMAC combines view-angle tilting and additional z-phase encoding, which provides information on how the slice profile is distorted, so that it can be corrected during image post-processing, thus eliminating both in-plane and through-plane distortions, with the counterpart of a longer scan time [7,9].

On a practical point of view, confronting acquisitions durations between STIR with view-angle tilting and STIR with SEMAC sequences, phantom, and in vivo results, the use of single view-angle tilting option could be a good compromise in clinical routine. Indeed, results obtained in vivo are comparable to what was observed in the phantom study, as STIR sequence coupled with view-angle tilting was the best fatsuppressed option. Adding SEMAC to the STIR sequence resulted in a much longer scan time, with little added value in terms of image quality. Complete MRI protocol applied to phantoms was too long (about $1 \mathrm{~h}, 40 \mathrm{~min}$ ) to be applied to patients: we therefore chose to include the sequences that had given the best results in the phantom study. Even if the Dixon technique seemed interesting experimentally, we decided not to evaluate it clinically because of the geometrical distortion it induced in the periacetabular region, and because of its incompatibility with MARS techniques.

Use of STIR with MARS may help to detect periprosthetic bone marrow edema and small pseudo-tumor, the first landmarks of serious conditions, which may not be well seen with other fat-suppression methods (FS, Dixon, SPAIR, WE) [20]. In our study, MARS-enhanced STIR allowed depiction of such abnormalities with good diagnosis confidence, which is 
Table 4 Clinical study, qualitative analysis for each patient

\begin{tabular}{|c|c|c|c|c|c|c|c|}
\hline \multirow[b]{2}{*}{ Patients } & \multicolumn{2}{|l|}{ STIR } & \multicolumn{2}{|l|}{ T2 FS VAT } & \multicolumn{2}{|l|}{ STIR VAT } & \multirow[b]{2}{*}{ Findings } \\
\hline & FSH & $\mathrm{DC}$ & FSH & $\mathrm{DC}$ & FSH & $\mathrm{DC}$ & \\
\hline 1 & 1 & 0 & 1 & 0 & 2 & 2 & None \\
\hline 2 & 1 & 1 & 0 & 0 & 2 & 2 & Intraarticular fluid \\
\hline 3 & 1 & 1 & 0 & 0 & 2 & 2 & None \\
\hline 4 & 1 & 1 & 0 & 0 & 2 & 2 & Intraarticular fluid \\
\hline 5 & 1 & 0 & 0 & 0 & 2 & 2 & Extraarticular collection \\
\hline 6 & 1 & 1 & 0 & 0 & 2 & 2 & None \\
\hline 7 & 1 & 1 & 0 & 0 & 2 & 2 & Intraarticular fluid \\
\hline 8 & 1 & 1 & 0 & 0 & 2 & 2 & Periprosthetic bone marrow edema \\
\hline Different from STIR & NA & NA & $p<0.0001^{*}$ & $p<0.0001^{*}$ & $p<0.0001^{*}$ & $p<0.0001^{*}$ & \\
\hline
\end{tabular}

*Indicates a statistically significant difference $(p<0.05)$

FSH fat suppression homogeneity, $D C$ diagnosis confidence

important to accurately orientate the surgeon to revision surgery.

The phantom used in this study aimed at modeling a human hip: agarose, a hydrophilic gel to simulate soft tissues and peanut oil to simulate subcutaneous and bone marrow fat. Peanut oil was chosen because its chemical compositionnotably the fat spectral peaks and $\mathrm{T} 1$ value - is close to those of human fat and it was used in several previous phantom studies [21-23]. Metal-on-metal total hip replacement implant was chosen among other wear couples, despite its decreasing use, because it is still present in a large population worldwide, and because liberation of metallic particles may induce pseudo-tumors that may require MRI for diagnosis. The results obtained in this study may be transposed to other wear couples with less metal burden.
The purpose of this study was not to compare fatsuppression techniques in terms of contrast or signal-tonoise ratio, but rather to determine the influence of metal on them. A ratio of contrast was used as the main variable, allowing quantitative analysis and comparison between different fat-suppression techniques. Our results are in line with the literature even if, to the best of our knowledge, no experimental study has yet evaluated the fat signal suppression around metallic orthopedic implants [24-26]. Le et al. have shown that artifacts induced by stainless-steel or titanium breast implants were greater in size with the FS technique than with the Dixon technique, focusing on the size of the artifact rather than on the fat-suppression homogeneity [24]. Zou et al. conducted an experimental study with different types of stainlesssteel and titanium plates at 1.5 T, including FS T2 and STIR,

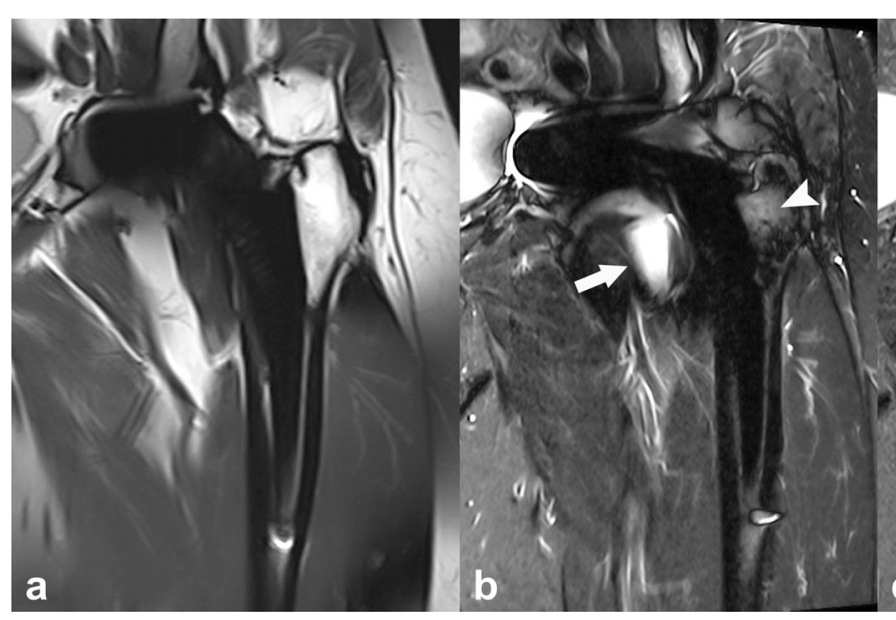

Fig. 4 Comparative coronal MR images of the right hip of a 71-year-old female patient: a TSE FS T2 with VAT, b STIR, c STIR with VAT, d STIR with SEMAC. T2 image shows heavy artifacts, precluding any reliable diagnosis. STIR image demonstrates areas of hypersignal below the

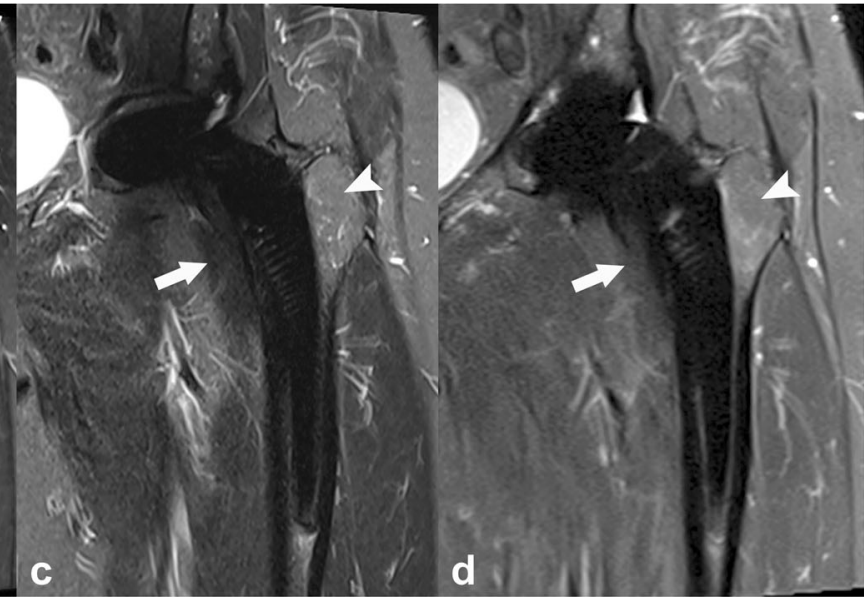

femoral implant neck (arrows) and in the great trochanter (arrowheads) that can be mistaken for intraarticular fluid and bone marrow edema. In contrast, both STIR-VAT and STIR-SEMAC sequences confirmed the absence of intraarticular fluid and normal bone marrow 


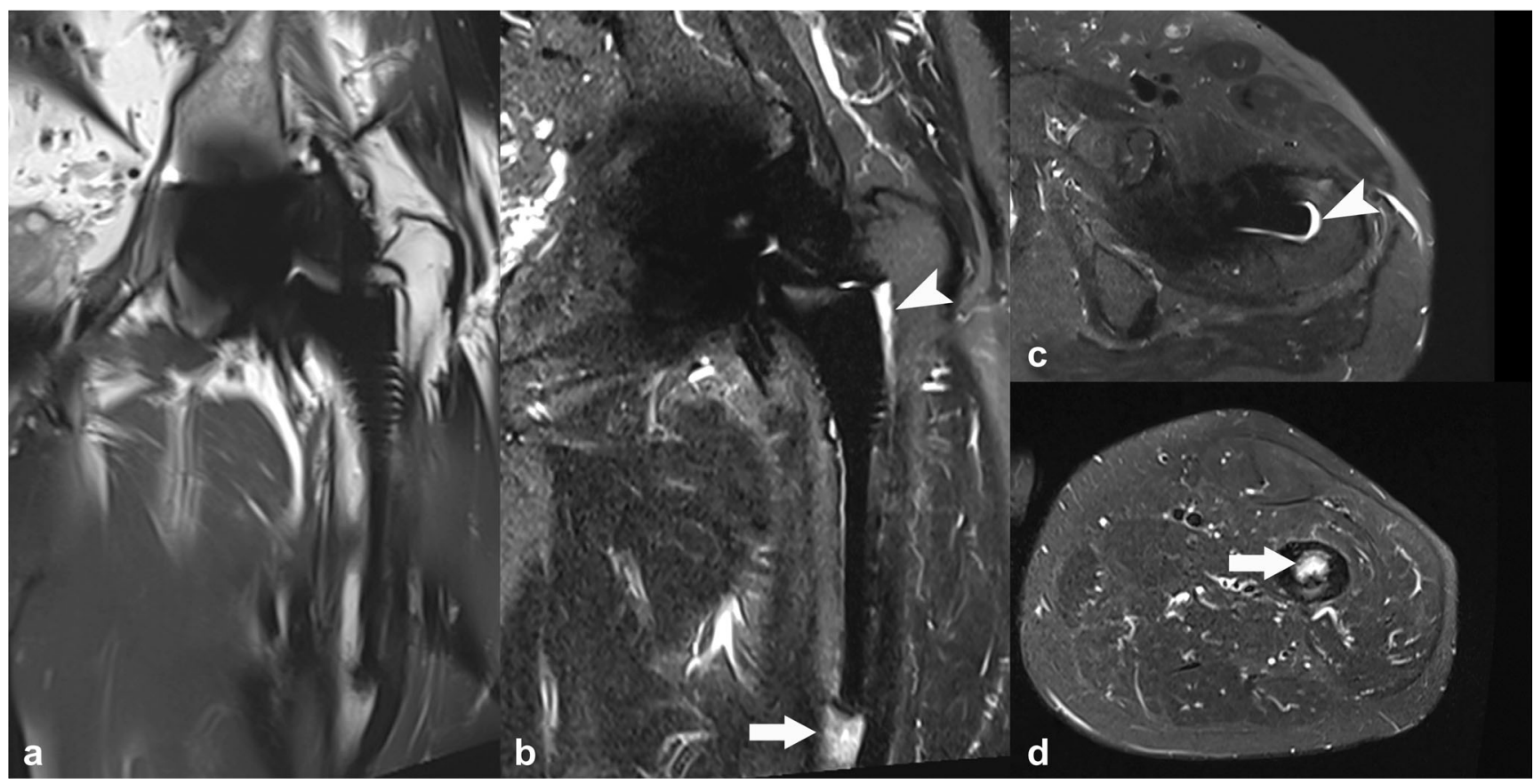

Fig. 5 MR images of the right hip of a 68-year-old male patient: a Coronal FS TSE T2 with VAT, b coronal STIR with VAT, $\mathbf{c}$ and $\mathbf{d}$ axial STIR with VAT. STIR VAT images nicely show periprosthetic femur

this last one giving a better result in terms of artifact size [25]. Winfield et al. compared performances of FS, STIR, and SPAIR techniques in large-FOV phantoms without metallic implant, finding that STIR provided the more homogeneous results [26]. The superiority of the STIR technique in the presence of metal have basic physical explanations. STIR is the only non-spectral fat-suppression method. It suppresses signals from tissues whose $\mathrm{T} 1$ is below a certain value, hence its low dependence on static magnetic field homogeneity [27].

This study was done on a 1.5-T MRI system. Even if numerous studies have demonstrated the abilities and advantages of 3-T imaging for the study of joints [28, 29], leading to an increased clinical use during the past 5 years, it should be taken into account that metal-induced artifacts greatly increase with $\mathrm{B}_{0}$ field, which could probably impact the fatsuppression quality $[29,30]$.

Our study had some limitations. First, clinical study was limited to a small population during a short period of time. We did not compare the accuracy of each technique for the detection of abnormalities around prosthesis, which would have required a larger cohort to include a significant set of pathologies, but we rather defined a diagnosis confidence score. Nevertheless, it could be reasonably thought that better fat suppression would allow better detection and characterization of abnormal findings. Last, we chose not to evaluate the Dixon technique in the clinical study because of severe periacetabular artifacts and the incompatibility of SEMAC with Dixon. Specific evaluation of T1-weighted imaging in a dedicated study could still bring interesting additional data. edema (arrow in b and d) as well as possible femoral implant loosening (arrowhead in $\mathbf{b}$ and $\mathbf{c}$ ) while T2 image is non diagnostic

In conclusion, STIR and Dixon are the preferred fluidsensitive MR sequences in patients with metal implants. Adding MARS to STIR significantly improves fat-suppression quality compared to other sequences. In particular, STIR with view-angle tilting seems to be the preferred sequence regarding the balance between acquisition time and fat-suppression quality.

\section{Compliance with ethical standards}

Conflict of interest The authors declare that they have no conflicts of interest.

\section{References}

1. Pivec R, Johnson AJ, Mears SC, Mont MA. Hip arthroplasty. Lancet Lond Engl. 2012;380:1768-77.

2.s Cushner F, Agnelli G, FitzGerald G, Warwick D. Complications and functional outcomes after total hip arthroplasty and total knee arthroplasty: results from the Global Orthopaedic Registry (GLORY). Am J Orthop Belle Mead NJ. 2010;39:22-8.

3. Koff MF, Shah P, Potter HG. Clinical implementation of MRI of joint arthroplasty. Am J Roentgenol. 2014;203:154-61.

4. Fritz J, Lurie B, Miller TT, Potter HG. MR imaging of hip arthroplasty implants. Radiogr. 2014;34:E106-32.

5. Hargreaves BA, Worters PW, Pauly KB, et al. Metal-induced artifacts in MRI. Am J Roentgenol. 2011;197:547-55.

6. Lüdeke KM, Röschmann P, Tischler R. Susceptibility artefacts in NMR imaging. Magn Reson Imaging. 1985;3:329-43.

7. Dillenseger JP, Molière $\mathrm{S}$, Choquet $\mathrm{P}$, et al. An illustrative review to understand and manage metal-induced artifacts in musculoskeletal MRI: a primer and updates. Skelet Radiol. 2016;45:677-88. 
8. Lee MJ, Janzen DL, Munk PL, et al. Quantitative assessment of an MR technique for reducing metal artifact: application to spin-echo imaging in a phantom. Skelet Radiol. 2001;30:398-401.

9. Lu W, Pauly KB, Gold GE, et al. SEMAC: slice encoding for metal artifact correction in MRI. Magn Reson Med. 2009;62:66-76.

10. Del Grande F, Santini F, Herzka DA, et al. Fat-suppression techniques for 3-T MR imaging of the musculoskeletal system. Radiogr. 2014;34:217-33.

11. Eggers H, Börnert P. Chemical shift encoding-based water-fat separation methods. J Magn Reson Imaging. 2014;40:251-68.

12. Bley TA, Wieben O, François CJ, Britain JH, Reeder SB. Fat and water magnetic resonance imaging. J Magn Reson Imaging JMRI. 2010;31:4-18.

13. Dixon WT. Simple proton spectroscopic imaging. Radiology. 1984;153:189-94.

14. Kolind SH, MacKay AL, Munk PL, et al. Quantitive evaluation of metal artifact reduction. J Magn Reson Imaging. 2004;20:487-95.

15. Jungmann PM, Ganter C, Schaeffeler CJ, et al. View-angle tilting and slice-encoding metal artifact correction for artifact reduction in MRI: experimental sequence optimization for orthopaedic tumor endoprostheses and clinical application. PLoS One. 2015;10: e0124922.

16. Choi S-J, Koch KM, Hargreaves BA, et al. Metal artifact reduction with MAVRIC SL at 3-T MRI in patients with hip arthroplasty. AJR Am J Roentgenol. 2015;204:140-7.

17. Talbot BS, Weinberg E. Imaging with metal-suppression sequences for evaluation of Total joint arthroplasty. Radiographics. 2016;36: $1-17$.

18. Oppelt A (2011) Imaging systems for medical diagnostics: fundamentals, technical solutions and applications for systems applying ionizing radiation, nuclear magnetic resonance and ultrasound. John Wiley \& Sons (p.214).

19. Landis JR, Koch GG. The measurement of observer agreement for categorical data. Biometrics. 1977;33:159-74.

20. Briant-Evans TW, Lyle N, Barbur S, et al. A longitudinal study of MARS MRI scanning of soft-tissue lesions around metal-on-metal total hip arthroplasties and disease progression. Bone Jt J. 2015;97B:1328-37.

21. Yu H, Shimakawa A, McKenzie CA, et al. Multiecho water-fat separation and simultaneous R2* estimation with multifrequency fat spectrum modeling. Magn Reson Med. 2008;60:1122-34.

22. Hess AT, Robson MD (2016) Hexagonal gradient scheme with RF spoiling improves spoiling performance for high-flip-angle fast gradient echo imaging. Magn Reson Med 0:1-7.

23. Hines $\mathrm{CDG}, \mathrm{Yu} \mathrm{H}$, Shimakawa $\mathrm{A}$, et al. $\mathrm{T} 1$ independent, $\mathrm{T} 2$ * corrected MRI with accurate spectral modeling for quantification of fat: validation in a fat-water-SPIO phantom. J Magn Reson Imaging JMRI. 2009;30:1215-22.

24. Le Y, Kipfer HD, Majidi SS, et al. Comparison of the artifacts caused by metallic implants in breast MRI using dual-echo Dixon versus conventional fat-suppression techniques. Am J Roentgenol. 2014;203:W307-14.

25. Zou Y, Chu B, Wang C, Hu Z. Evaluation of MR issues for the latest standard brands of orthopedic metal implants: plates and screws. Eur J Radiol. 2015;84:450-7.

26. Winfield JM, Douglas NHM, Desouza NM, Collins DJ. Phantom for assessment of fat suppression in large field-of-view diffusionweighted magnetic resonance imaging. Phys Med Biol. 2014;59: 2235-48.

27. Ulbrich EJ, Sutter R, Aguiar RF, et al. STIR sequence with increased receiver bandwidth of the inversion pulse for reduction of metallic artifacts. Am J Roentgenol. 2012;199:W735-42.

28. Ramnath RR. 3T MR imaging of the musculoskeletal system (part II): clinical applications. Magn Reson Imaging Clin N Am. 2006;14:41-62.

29. Lee M, Kim S, Song H, et al. Overcoming artifacts from metallic orthopedic implants at high-field-strength MR imaging and multidetector CT. Radiographics. 2007;27:791-804.

30. Shapiro L, Harish M, Hargreaves B, et al. Advances in musculoskeletal MRI: technical considerations. J Magn Reson Imaging JMRI. 2012;36:775-87. 